Service social

\title{
Entraide et service social des groupes
}

\section{Estelle Hopmeyer}

Volume 39, numéro 1, 1990

Le groupe ici et ailleurs

URI : https://id.erudit.org/iderudit/706457ar

DOI : https://doi.org/10.7202/706457ar

Aller au sommaire du numéro

Éditeur(s)

École de service social de l'Université Laval

ISSN

1708-1734 (numérique)

Découvrir la revue

Citer cet article

Hopmeyer, E. (1990). Entraide et service social des groupes. Service social, 39(1), 64-74. https://doi.org/10.7202/706457ar
Résumé de l'article

Le présent article examine cinq des caractéristiques propres à la structure des groupes d'entraide, propose des orientations d'intervention à l'intention des professionnels qui désirent apporter leur soutien à ce type de groupes, et présente brièvement quelques-unes des limites ou difficultés associées à cette forme d'aide. d'utilisation que vous pouvez consulter en ligne.

https://apropos.erudit.org/fr/usagers/politique-dutilisation/ 


\title{
Entraide et service social des groupes*
}

\author{
Estelle Hopmeyer
}

Les groupes de service social ont été animés par des travailleurs sociaux professionnels qui facilitaient l'apparition et le développement du processus d'aide mutuelle. Nous constatons une nouvelle émergence, durant les deux dernières décennies, des groupes d'entraide; ceux-ci se différencient des groupes de service social en ce qu'ils sont organisés, dirigés et contrôlés d'abord par leurs membres. Ces groupes reposent sur le postulat que ceux qui ont expérimenté une situation particulière de vie sont les plus compétents sur ce sujet (Home, $1985: 73$ ).

La documentation récente suggère que l'entraide peut suppléer à l'aide apportée par les professionnels et que la connaissance acquise par l'expérience d'une part, la compétence et les habiletés professionnelles d'autre part, sont complémentaires (Biegel et Naparstek, 1982; Lieberman et al., 1979). Gottlieb (1982) a constaté que les membres des groupes d'entraide ne voient pas de conflit important entre l'aide professionnelle et l'entraide et, en fait, certains utilisent les deux types d'aide. De plus, les écrits font mention de toute une variété de rôles que les professionnels jouent en relation avec les groupes d'entraide (Coplon et Strull, 1983; Maguire, 1984; Silverman, 1980; Toseland et Hacker, 1982). Cette même documentation invite les professionnels à adhérer à la philosophie de l'entraide et à favoriser cette forme d'aide (Whittaker et Garbarino, 1983; Gottlieb, 1988). Le présent article examinera cinq des caractéristiques propres à la structure des groupes d'entraide et proposera des orientations d'intervention à l'intention des professionnels qui désirent apporter leur soutien à ce type de groupes. Enfin, il présentera brièvement quelques-unes des limites ou difficultés associées à cette forme d'aide. 


\section{Groupes d'entraide}

L'entraide est fondée sur une philosophie qui reconnaît la force des gens, la développe et la nourrit, non seulement pour les amener à s'aider eux-mêmes, mais aussi pour les inciter à rejoindre les autres et à les aider à s'aider eux-mêmes (Gottlieb, 1988). L'entraide s'adresse à l'ensemble de la population et ne dépend pas des priorités des services gouvernementaux ou institutionnels. Elle ne subit pas de contraintes de temps, ni de lieu, ni de formule. L'entraide est gratuite. Elle n'est pas à la remorque des professionnels, des institutions ou des agences officielles, mais focalise plutôt sur l'éducation et sur le soutien entre pairs. La différence entre le rôle des membres du groupe et celui du praticien est réduite au minimum puisque l'entraide est basée sur le partage de l'expérience plutôt que sur une formation institutionnelle. L'entraide met l'accent sur une approche de réseau ou de groupe et peut aussi chercher l'appui de personnes significatives telles que des partenaires ou des proches qui prennent soin du membre.

Un groupe d'entraide peut être défini comme un petit groupe bénévole formé de gens qui partagent une condition, un intérêt ou une expérience commune et qui se réunissent dans le but de s'aider mutuellement (Lieberman et al., 1979). La structure du groupe d'entraide est différente de celle du groupe de soutien (Gottlieb, 1988) des cinq façons suivantes ${ }^{2}$ :

1. Les groupes de soutien sont dirigés et sanctionnés par des professionnels, alors que les groupes d'entraide le sont par leurs membres.

2. Les groupes de soutien combinent la connaissance professionnelle et la connaissance issue de l'expérience alors que les groupes d'entraide reposent principalement sur la connaissance issue de l'expérience.

3. Les groupes de soutien ont généralement une limite de temps tandis que les groupes d'entraide n'en ont pas.

4. Les groupes de soutien réunissent généralement les mêmes membres durant toute la durée du groupe tandis que les groupes d'entraide acceptent de nouveaux membres en tout temps.

5. En général, les groupes de soutien ne s'engagent pas dans des actions sociales, des mouvements de pression, de défense des droits ou d'éducation communautaire, tandis que plusieurs groupes d'entraide considèrent que ces activités font partie de leur mandat.

En examinant ces caractéristiques, nous pouvons identifier des conséquences qui en découlent pour la pratique professionnelle et 
suggérer quelques pistes aux intervenants qui désirent s'engager dans cette forme d'aide.

\section{Direction assurée par les membres}

Les groupes d'entraide ne laissent pas tous une place égale au professionnel. Ils se situent plutôt sur un continuum où on retrouve, à un extrême, l'absence de participation du professionnel (groupes anonymes) et, à l'autre extrême, un professionnel qui tient lieu de répondant (parents abusifs). Toutefois, même dans cette dernière situation, le professionnel agit à titre de conseiller et les membres du groupe ne sont pas considérés comme des clients qui ont besoin de traitement. La fonction principale du professionnel est de suppléer aux ressources des membres, particulièrement à celles des responsables (leaders). Plus précisément, les écrits mentionnent quelques rôles joués par les professionnels auprès des groupes d'entraide : catalyseur, assistant technique, consultant ou personne-ressource (Coplon et Strull, 1983; Gartner et Reissman, 1977; Harris, 1981; Maguire, 1984; Silverman, 1980).

\section{A. Catalyseur}

Le professionnel agit comme catalyseur lorsqu'il aide à la formation du groupe en évaluant ses besoins, en recrutant des membres potentiels, en fournissant des ressources concrètes comme une salle de réunion, du café ou du gardiennage, et en assurant la liaison avec d'autres groupes ou organisations. Le praticien peut faire face à une résistance de la part de collègues et d'autres professionnels, peut avoir à agir comme tampon, à donner de l'information et à légitimer la formule du groupe d'entraide en s'appuyant sur sa propre crédibilité professionnelle. À cette étape, le catalyseur doit encourager des membres potentiels à collaborer activement de façon à s'assurer qu'ils prendront le groupe en mains dès les débuts.

À ce stade, une partie de son travail consiste à établir un contrat avec le groupe quant à son implication future. À la différence des formes d'intervention de groupe plus traditionnelles, le professionnel peut planifier de se retirer très tôt, peut-être même après la première rencontre dans certains cas. Si la durée du contrat initial est plus longue, il est essentiel de prévoir un mécanisme de révision et d'évaluation continues de la participation du professionnel et de son rôle auprès du groupe, évitant ainsi qu'il garde la direction du groupe au-delà du temps nécessaire. Ceci contribuera également à contrer la résistance du groupe à renoncer au soutien professionnel. 


\section{B. Assistant-consultant technique ou personne-ressource}

Après le démarrage du groupe, le professionnel peut se retirer tout en restant disponible sur appel comme personne-ressource ou comme consultant. II pourrait s'agir, par exemple, d'un suivi de formation auprès du responsable du groupe. Comme le suggère Silverman (1980), le travailleur social peut enseigner aux membres à se sentir à l'aise dans le processus d'aide mutuelle sans toutefois chercher à en faire deś professionnels. Si l'intervenant reste présent aux réunions de groupe, il devrait assumer volontairement un rôle périphérique, de façon à ce que le processus d'aide puisse prendre racine au sein du groupe, basé sur la connaissance que les membres ont acquise par l'expérience.

Néanmoins, il est parfois nécessaire d'intervenir plus activement dans le processus de groupe. Fidèle à la philosophie de l'entraide, le professionnel devrait limiter ses interventions aux moments où le groupe demande ou requiert une information pertinente ou lorsqu'il apparaît clairement qu'une situation risque d'être nuisible à une personne ou à l'ensemble du groupe. La notion de "nuisible " peut être définie à l'intérieur du contrat établi entre le groupe et l'intervenant ou encore se fonder sur le jugement éthique du professionnel. Ce dernier peut intervenir pour éviter qu'une personne très vulnérable ne soit questionnée prématurément, pour faire le contrepoids à un point de vue très catégorique (par exemple, une recommandation contraire au traitement, qui risque d'être dommageable dans un groupe d'entraide axé sur des problèmes médicaux) ou pour soulever une question d'éthique (ne pas dévoiler à quelqu'un que son partenaire sexuel est porteur du virus du sida, par exemple). Cependant, il est essentiel que le professionnel ne devienne pas une figure centrale dans le groupe lorsqu'il donne de l'information ou qu'il fait profiter le groupe de son expertise et de sa compétence.

II est aussi important de souligner le recours volontairement limité à l'intervention professionnelle. Les groupes d'entraide diffèrent en cela de la majorité des groupes traditionnels dans lesquels l'intervenant utilise sa connaissance et sa compétence pour faciliter le processus de groupe et, par moments, pour aider le groupe à prendre des décisions plus éclairées. Rester à l'écart n'est pas une tâche facile pour l'intervenant car il arrive parfois que le responsable du groupe soit incapable de prendre une situation en mains. Ceci peut se produire, par exemple, lorsqu'il est confronté à une émotion forte telle que la colère, ou encore lorsqu'il est aux prises avec un membre qui parle tout le temps. Le professionnel doit être prêt à voir le groupe lutter et, parfois, essuyer des échecs à court terme pour enfin 
atteindre l'objectif à long terme qu'est l'accroissement du pouvoir et de l'autonomie des membres.

\section{Rôles indirects}

Les professionnels jouent toute une variété de rôles plus indirects auprès des groupes d'entraide. Ils sont membres de comités consultatifs, dirigent des clients vers des groupes d'entraide et continuent à "éduquer" leurs collègues et d'autres professionnels quant à la valeur de l'aide naturelle (non professionnelle). Pour cela, ils doivent comprendre qu'il n'existe pas de contradiction en soi entre l'aide professionnelle et l'aide naturelle. En plus d'offrir une aide individualisée (Whittaker et Garbarino, 1983), les professionnels doivent considérer le contexte social comme partie intégrante de l'aide interpersonnelle, et travailler à consolider ou à établir des formes de soutien social pour les individus.

\section{Connaissance acquise par l'expérience}

La connaissance acquise par l'expérience est à la base de l'entraide. Les écrits identifient plusieurs activités et processus qui rendent cette forme d'aide particulièrment efficace (Biegel et Naparstek, 1982; Caplan et Killilea, 1976; Gartner et Reissman, 1977; Lieberman et al., 1979; Reissman, 1976). Nous en ferons un bref survol ici pour faire ressortir des façons dont les professionnels peuvent contribuer à ces processus. (Une discussion plus complète de cette question est présentée dans l'étude de Claude Olivier (1989) sur le travail de groupe avec des personnes séropositives).

Les processus qui, croit-on, contribuent à l'efficacité du modèle de l'entraide peuvent être mesurés de deux façons. La première s'intéresse aux activités des membres dans leurs interactions visant à donner et à recevoir de l'aide, tandis que l'autre met l'accent sur les processus qui découlent de ces activités (Lieberman et al., 1979).

Levy a relevé les phénomènes les plus fréquemment observés à I'intérieur des groupes d'entraide (Lieberman et al., 1979 : 260-261). Plusieurs exemples rapportés comportent de l'empathie, de l'encouragement (on amène quelqu'un à croire que ses problèmes vont éventuellement se régler d'une façon positive) et des explications (on aide d'autres membres à mieux se comprendre eux-mêmes et à mieux saisir leur situation). Les membres encouragent souvent d'autres membres à se livrer, à partager et à s'affirmer.

Certains écrits dont ceux de Katz (cité dans Gartner et Reissman, 1977), ainsi que les travaux en cours de l'auteure auprès d'un groupe d'entraide constitué de personnes qui ont vécu le suicide d'un membre de leur famille, le FSOS (Family Survivors of Suicide) ${ }^{3}$, 
identifient aussi des processus associés à l'efficacité de l'entraide. Deux d'entre eux seront présentés ici : la réciprocité de l'aide et l'expansion du réseau social. La réciprocité de l'aide est au cœur même du processus d'aide mutuelle. Frank Reissman (1976) a décrit différentes façons par lesquelles un membre qui apporte de l'aide est aidé lui aussi; bien sûr, il a constaté qu'il s'agissait là d'une des composantes les plus importantes de l'entraide. En aidant les autres, les membres ont un sentiment accru d'utilité sociale et de compétence interpersonnelle, ce qui rehausse leur estime d'eux-mêmes. L'aide apportée dépend de la connaissance qu'ils ont acquise par l'expérience et prend différentes formes selon le style de chacun. Reissman (1976) a appelé cette composante de l'entraide la " dimension aprofessionnelle » et a remarqué qu'elle se démarquait d'une approche professionnelle en ce que l'aide apportée tend à être plus subjective, plus informelle, plus concrète, plus pertinente dans l'immédiat et plus accessible.

L'expansion du réseau social est une autre conséquence des groupes d'entraide dont bénéficient les membres (Kurtz et Powell, 1987). Le groupe peut faire connaître aux membres d'autres personnes à qui ils peuvent s'identifier et qui peuvent servir de points de référence ou de comparaison sociale. Bien des gens arrivent dans un groupe d'entraide en se sentant seuls avec leurs problèmes. Ils ont souvent l'impression d'être inadéquats parce qu'ils ont un problème ou parce qu'ils ne sont pas capables d'y faire face. La rencontre de personnes qui sont " dans la même galère ", fait souvent en sorte que leurs sentiments d'incompétence et d'échec peuvent faire place à un sentiment de normalité dans les circonstances. De plus, les membres peuvent sentir qu'ils risquent moins d'être jugés ou mal compris puisque d'autres membres partagent les mêmes expériences ou des expériences similaires. Le soutien développé à l'intérieur des groupes peut aussi se prolonger au-delà des réunions; par exemple, des membres peuvent échanger leurs numéros de téléphone et se rencontrer à d'autres moments.

Pour comprendre le processus d'aide mutuelle, examinons quelques exemples d'échanges entre les membres ${ }^{4}$.

Le père et la mère d'une jeune fille qui venait de se suicider sont restés silencieux pendant presque toute la première réunion à laquelle ils ont assisté. À la toute fin, la mère a raconté leur histoire, exprimant énormément de culpabilité face à la mort de leur fille parce qu'ils n'avaient pas compris la profondeur réelle de son désespoir et ne l'avaient pas hospitalisée. Dans le silence qui a suivi, un autre père a dit : "Vous aimiez votre fille et elle vous aimait. Vous êtes de très bons parents ». Au cours de la réunion suivante, la mère dit que, pour la première fois depuis le suicide, elle s'était. sentie bien pendant quatre heures le jour suivant. Plusieurs mois 
plus tard, cette même mère assistait à une rencontre où un nouveau membre racontait au groupe qu'elle avait eu de la difficulté à obtenir des soins psychiatriques et que, finalement, elle s'était rendue à l'hôpital pour voir un psychiatre. Après cette entrevue initiale, le médecin a recommandé qu'elle soit immédiatement hospitalisée. Elle a accepté à contrecœur mais lorsqu'elle est arrivée à l'unité, elle a refusé de rester à cause de l'atmosphère et des patients qui étaient là. Pendant qu'elle racontait cette expérience, la première mère écoutait attentivement et, quand le récit a été terminé, elle lui a dit à quel point cela lui avait fait du bien. Elle se rendait maintenant compte que I'hospitalisation n'aurait pas nécessairement sauvé sa fille.

Ces échanges basés sur la connaissance acquise par l'expérience peuvent être très aidants. Le rôle du professionnel consiste à aider le responsable du groupe à créer un climat d'ouverture, d'acceptation, où les membres ne seront pas jugés. II est parfois difficile d'assurer l'équilibre entre le désespoir d'une part, et l'inspiration et l'espoir d'autre part, comme par exemple dans les groupes de victimes du sida ou du cancer.

\section{Groupes continus et ouverts}

Les groupes d'entraide sont ouverts, c'est-à-dire continuellement accessibles à de nouveaux membres, permettant de répondre aux besoins d'une façon continue et permettant à chacun de décider de sa propre participation (Galinsky et Schopler, 1985). Les problèmes associés à cette formule n'appartiennent pas qu'aux groupes d'entraide. Le processus de groupe peut être perturbé par l'arrivée de nouveaux membres ou par le départ ou l'absence d'un noyau de membres. Ceux qui restent peuvent éprouver des sentiments de perte, d'envie ou d'abandon. Enfin, des changements fréquents dans la composition du groupe impliquent que les membres ne passent pas assez de temps ensemble pour qu'émergent vraiment des leaders susceptibles d'assumer la responsabilité du groupe.

Le professionnel qui travaille avec un groupe d'entraide ouvert fait face à un dilemme, celui de déterminer dans quelle mesure il doit participer à l'insertion d'un membre dans le groupe ou à son départ. Coplon et Strull (1983) suggèrent une participation active de l'intervenant à toutes les étapes de développement du groupe, à partir de la pré-affiliation et de l'établissement du contrat jusqu'à la fin, lorsque les membres sont aidés à s'engager dans une nouvelle expérience. Cette contribution apparaît plus appropriée auprès de groupes de soutien que de groupes d'entraide. Dans le cas de ces derniers, la notion de "nuisible» (pour une personne ou pour l'ensemble du 
groupe), discutée plus tôt, devrait aider à déterminer le degré de participation du professionnel. Comme dans plusieurs cas il n'y a pas de rencontre préliminaire avec les membres potentiels, il se présente quelquefois des personnes avec lesquelles le groupe ne peut pas composer. Si l'intervenant est présent, il peut rencontrer ces personnes à l'extérieur du groupe et les orienter vers une autre ressource ou alors, s'il y a lieu, aider le responsable à les intégrer au groupe par "modelling", de façon à ce qu'ils ne se sentent pas rejetés. Le professionnel peut aussi aider le groupe à développer des mécanismes pour assurer un suivi aux membres absents, ou aider le responsable à animer des discussions à propos des sentiments de perte ou d'abandon vécus à la mort d'un membre, comme cela arrive dans des groupes dont les membres présentent des problèmes médicaux ou lors du départ d'une personne qui quitte en conflit avec le groupe.

\section{Action sociale, défense des droits, éducation communautaire}

Les groupes d'entraide ne s'engagent pas tous au même degré dans des actions sociales, des activités de défense des droits, etc. À une extrémité de ce continuum, on retrouve les groupes anonymes qui ne s'engagent pas dans de telles activités (quoiqu'ils s'intéressent activement à l'éducation du public) et, à l'autre extrême, des groupes tels que celui de mères qui luttent contre l'alcool au volant : "Mothers Against Drunk Drivers» (MADD), pour qui l'action sociale est la principale raison d'être. Plusieurs autres groupes inscrivent dans leurs mandats certains aspects de défense des droits ou d'éducation du public. Claude Olivier (1989 : 24) note que la défense des droits peut être un élément de travail important pour des groupes opprimés, tels les porteurs du virus du sida, souvent marginalisés en raison de leur maladie ou de leur orientation sexuelle. II cite ainsi un membre du groupe :

Je voudrais voir [sic] plus de pressions politiques, dans ce sens que les gens encouragent les levées de fonds, s'intéressent davantage au logement, aux médicaments, à la recherche. Cette mobilisation est importante non seulement parce que je crois qu'il est essentiel d'atteindre ces buts, mais aussi pour nous permettre de relâcher la tension et l'anxiété provoquées par le fait d'être confrontés à un virus contre lequel nous ne pouvons à peu près rien.

Ces processus accroissent généralement le pouvoir des gens et le professionnel devrait encourager les initiatives que le groupe désire entreprendre dans ce sens. Ainsi, il peut aider les membres à travailler avec les médias et avec d'autres ressources, leur apporter informa- 
tions et connaissances sur les processus politiques. Cependant, le professionnel devrait garder à l'esprit les risques possibles de telles activités. Premièrement, elles peuvent engager le groupe dans une démarche d'analyse politique (reliant l'expérience personnelle à des processus structuraux plus larges) qui peuvent non seulement mobiliser les membres mais aussi les diviser. Deuxièmement, elles peuvent ne pas correspondre au système de valeurs du professionnel ou à son expertise et, parfois, mettre ce dernier en conflit avec un employeur. Cette difficulté n'appartient pas qu'aux groupes d'entraide.

\section{Limites des groupes d'entraide}

Les auteurs ont largement documenté les avantages du soutien social et de l'entraide (Biegel et Naparstek, 1982; Gartner et Reissman, 1977; Gottlieb, 1988; Lieberman et al., 1979; Nicholaichuk et Wollert, 1980; Whittaker et Garbarino, 1983). Toutefois, cette forme d'aide peut aussi présenter des dangers. Le professionnel doit connaître ces limites de façon à maximiser les effets positifs décrits plus tôt. Voici un bref aperçu de ces principales limites.

1. L'entraide peut contribuer à privatiser des services qui devraient être financés par l'État (par exemple, des groupes de soins, qui sont principalement composés de femmes), malgré que l'action sociale puisse tenir une place importante dans ces groupes.

2. Les groupes d'entraide peuvent, d'une certaine façon, contribuer à " blâmer les victimes ", puisque les participants font du problème leur principale identité. Ceci peut encourager une dépendance prolongée ou inappropriée.

3. Les groupes d'entraide peuvent être une solution de rechange inappropriée à l'aide professionnelle. Des membres peuvent participer sans être aidés. Ces groupes peuvent omettre d'établir et d'évaluer leurs objectifs, étape qui constitue une partie essentielle de la pratique professionnelle.

4. Les groupes d'entraide peuvent avoir une forte tendance à être autoritaires, en raison soit de leur idéologie, soit de leurs leaders. Ils peuvent devenir fermés et intolérants devant les différences individuelles.

5. Les groupes d'entraide peuvent être dirigés en partie par des professionnels ou par des agences qui restreignent l'autonomie du groupe. 


\section{Conclusion}

Les groupes d'entraide ont beaucoup en commun avec les autres formes de service social des groupes, principalement en ce qui a trait aux processus d'aide mutuelle; toutefois, ils présentent aussi des différences importantes en termes de philosophie et de pratique, particulièrement en ce qui concerne le rôle et la fonction des professionnels. Les intervenants qui travaillent avec les groupes d'entraide doivent croire sincèrement au pouvoir de la connaissance acquise par l'expérience et à l'aide apportée par les pairs, et être prêts à limiter vraiment leur intervention auprès du groupe. Ceci ne doit pas être perçu comme un refus de partager leurs connaissances et leurs compétences professionnelles, mais bien comme une façon spéciale d'utiliser leur propre personne. David Soyer, dans son article intitulé "The Right to Fail », exprimait très bien ceci lorsqu'il disait :

"Nous faisons appel à plus d'audace de la part des travailleurs sociaux, impliquant une alliance plus grande avec les aspirations des clients, même quand celles-ci apparaissent hors d'atteinte et comportent un risque d'échec. Le droit à l'autodétermination passe par le droit d'échouer... » (Soyer, $1963: 78$ ).

Le travail auprès de groupes d'entraide peut aider les professionnels à « reconceptualiser " leur rôle dans la pratique du service social des groupes et à développer un modèle qui favorise le plus d'autonomie possible chez les membres. Ceci accroît le pouvoir non seulement des membres, mais aussi des professionnels.

\section{Notes}

* Texte traduit par Lyne Champoux.

- L'auteure désire remercier Claude Olivier pour sa collaboration dans la préparation de cet article dont le contenu a été discuté dans le cadre des "déjeuners par groupes d'intérêts »-lors du symposium de Montréal.

2 Avec des modifications mineures, cette comparaison peut s'appliquer à plusieurs autres formes de petits groupes, tels que les groupes d'éducation, de traitement et ceux issus du courant central.

3 Le groupe FSOS a été mis sur pied par une mère qui, après le suicide de sa fille en novembre 1987, n'a pu trouver de groupe de soutien pour des familles qui ont vécu un suicide. Le groupe est dirigé par ses membres, et une professeure de l'Ecole de service social de l'Université McGill agit à titre de consultante.

${ }^{4}$ Ces exemples proviennent de deux rencontres du FSOS.

\section{Références}

Biegel, D.C. et A. NAPARStek (1982). Community Support Systems and Mental Health : Practice, Policy and Research. New York: Springer Publishing Company. 
BORKMAN, T. (1976). "Experiential Knowledge : A New Concept for the Analysis of Self-Help Groups", Social Service Review, vol. 50 (septembre) : 446-456.

Caplan, G. et M. Killilea (1976). Support Systems and Mutual Help : Multidisciplinary Exploration. New York : Gruner and Stratton.

COPLON, J. et J. Strull (1983). "Roles of the Professional in Self-Help Groups", Social Casework, vol. 64, $\mathrm{n}^{\circ} 5$ : 259-266.

Galinsky, M. et J. SChOPLeR (1985). "Patterns of entry and exit in open-ended groups ", Social Work with Groups, vol. 8, $\mathrm{n}^{\circ} 2$ : 67-80.

Gartner, A. et F. Reissman (1977). Self-Help in the Human Services. San Francisco : Jossey-Bass.

GotmLleB, B.H. (1982). Prevention in the Social Services, vol. 1, $n^{\circ}$ 3. California : Sage Publications.

Gottueb, B.H. (éd.) (1988). Marschalling Social Support: Formats, Processes and Effects. Newbury Park : Sage Publications.

HaRrIS, Z. (1981). "Ten Steps to Establishing a Self-Help Group ", Canada's Mental Health, vol. 29, $\mathrm{n}^{\circ} 1: 16$

HOME, A. (1985). "Intervention With Groups ": 69-89, dans S.A. Yelaga, An Introduction to Social Work Practice in Canada. Scarborough, Ontario : Prentice-Hall Canada Inc.

KATZ, A.H. (1970). "Self-Help Organizations and Volunteer Participation in Social Welfare ", Social Work, vol. $15:$ 51-60.

KURTZ, L. et T. POWELL (1987). "Three Approaches to Understanding Self-Help Groups ", Social Work with Groups, vol. 10, $\mathrm{n}^{\circ} 3: 69-80$.

LeVy, L.H. (1979). "Processes and Activities in Groups": 234-271, dans M.A. Lieberman et L.D. Borman (éd.), Self-Help Groups for Coping with Crisis. San Francisco : Jossey-Bass.

Lieberman, M., L.D. Borman et associés (1979). Self-Help Groups for Coping with Crisis. San Francisco : Jossey-Bass.

MAGUIRE, L. (1984). "Networking for Self-Help : An Empirically Based Guideline ": 198-209, dans F. Cox, J.L. Erlich, J. Rothman, J.E. Tropman, Tactics and Techniques of Community Practice. Itasca, III. : F.E. Peacock Publishers.

Nicholaichuk, T.P. et R. WOllert (1989). "The Effects of Self-Help on Health Status and Health Services Utilization ", Canadian Journal of Community Mental Health, vol. 8, $\mathrm{n}^{\circ} 1: 17-29$.

Olivier, Claude (1989). Group Work with People with HIV Infection. Mémoire de maîtrise, McGill University.

Reissman, F. (1976). "The Helper Therapy Principle", Social Work, vol. 10 : 27-32.

Silverman, P. (1980). Mutual Health Groups - Organization and Development. California : Sage Publications.

SOYER, D.S. (1963). "The Right to Fail ", Social Work, vol. 8, no 3 : 72-78.

TOSELAND, R.W. et L. HACKER (1982). "Self-Help Groups and Professional Involvement ", Social Work, vol. 27, $\mathrm{n}^{\circ} 4: 341$.

Whittaker, J.K., J. Garbarino et associés (1983). Social Support Networks: Informal Helping in the Human Services. New York : Aldine du Gruyter. 